\title{
Research on EMI Noise Measurement Technology Based on Wavelet Analysis
}

Xu Li The First Affiliated Hospital of Hebei North University, Zhang Jiakou, Hebei, $07500 \quad 15933243301$

Long Zaiyun Hebei University of Architecture, Zhang Jiakou, Hebei, 0750015931392613

\begin{abstract}
Aiming at the problem that it is difficult to measure the electromagnetic radiation produced by the equipment at present, this paper presents a method for measuring the noise of electromagnetic interference (EMI) based on wavelet analysis. The technique uses time frequency localization features of the wavelet transform, based on threshold function filtering method to filter the test signal, which makes it possible in open space or noisy environment for measurement of electromagnetic interference of equipment. Simulation and experimental results show that the technique is able to eliminate or attenuate the noise in the frequency band of $30 \mathrm{~Hz} 1000 \mathrm{MHz}$.
\end{abstract}

Key words: Electromagnetic interference (EMI) measurement; wavelet analysis; frequency division filter; threshold function

\section{Published Online: 31st Jan 2018}

\section{Introduction}

In today's society, with the continuous development of electronic information technology, the application of electronic equipment is more and more widespread, and many electronic components contained in it can cause electromagnetic interference, such as electrostatic discharge and surge, seriously affecting people's work and life. Therefore, how to measure the electromagnetic radiation generated by a device rapidly and accurately is an urgent problem to be solved. When measuring the electromagnetic interference of a device, it can be found that the signal acquired contains many noises due to the radiation interference from surrounding devices or mobile communications and the ionosphere. Therefore, at present, electromagnetic interference measurement of devices needs to be performed in a confined space such as a dark room or in an empty open space ${ }^{[1-5]}$.

Aiming at the problem that it is difficult to accurately measure the electromagnetic radiation produced by the equipment, this paper presents a method for measuring the noise of electromagnetic interference (EMI) based on wavelet analysis. The technique uses time frequency localization features of the wavelet transform, based on threshold function filtering method to filter the test signal, which makes it possible to measure electromagnetic interference of equipment in an open area or noisy environment.

\section{Wavelet analysis}

\subsection{Wavelet transform}

The wavelet transform can be shown as equation (1).

$$
\mathrm{WT}_{x}(a, \tau)=\frac{1}{\sqrt{a}} \int_{-\infty}^{+\infty} x(t) \Psi^{*}\left(\frac{t-\tau}{a}\right) \mathrm{d} t, \quad a>0
$$

$\Psi(t)$ is the wavelet function, and $a$ is the scale, which makes the resolution higher with the frequency increases.

Because Morlet wavelet has the real-time performance when it processes signal, this paper chooses it to perform wavelet transform, as (2), (3).

$$
\begin{aligned}
& \psi(t)=e^{-t^{2} / 2} e^{h_{\omega_{0} t}} \\
& \widehat{\psi}(\omega)=\sqrt{2 \pi} e^{-\left(\omega-\omega_{0}\right)^{2} / 2}
\end{aligned}
$$

Discrete wavelet transform is performed by the discretization of wavelets function, as (4), (5).

$$
\begin{aligned}
& \psi_{j, k}(t)=2^{j / 2} \psi\left(2^{j} t-k\right) \\
& W_{f}(j, k)=\left(f(t), \psi_{j . k}(t)\right)
\end{aligned}
$$




\subsection{Construction of threshold function}

Since the energy of the useful signal is concentrated in a larger wavelet coefficient while the energy of the noise is distributed in the entire wavelet domain, and the energy amplitude of the signal is larger than that of the noise energy after the wavelet decomposition ${ }^{[6]}$, so the principle of the threshold function is that all the wavelet coefficients are retained when the resolution is low, and the wavelet coefficients above the threshold are reserved by setting the threshold when the resolution is high. And the reconstruction of signal is performed in the end. The filtering method in wavelet transform can be divided into soft threshold denoising and hard threshold denoising ${ }^{[7]}$. Combined with the advantages of good continuity of soft threshold denoising signal and small denoising error of hard threshold ${ }^{[8]}$, the threshold function is constructed, as follows:

$$
\begin{gathered}
W_{0}(x)=\operatorname{sgn}(x)\left(|x|-\frac{b \lambda}{e^{(\mid(x \mid-\lambda) / \lambda)}}\right),|x| \geq \lambda \\
\begin{array}{c}
W(x)=0, \quad|x<\lambda| \\
\lambda=\sqrt{2 \log (N) \sigma}
\end{array}
\end{gathered}
$$

When $x>0$, the equation (6) becomes

$$
\frac{W(x)}{x}=1-\frac{b \lambda}{x e^{((x-\lambda) / \lambda)}}(7)
$$

When $\mathrm{x}<0$, the equation (7) becomes

$$
\frac{w(x)}{x}=1+\frac{b \lambda}{x e^{((-x-\lambda) / \lambda)}}
$$

The traditional Fourier transform can not show the changes of signal within a certain period of time, which means no sudden changes or transient phenomenon can be identified. Once the signal changes, the entire spectrum will be affected. While the wavelet transform can analyze the signal in time domain and frequency domain, and reflect the local variation of signal, which is especially suitable for analyzing non-stationary signal. In MATLAB, the signal is processed by using Fourier transform and wavelet transform respectively, and the comparison figures are shown in Figure1.

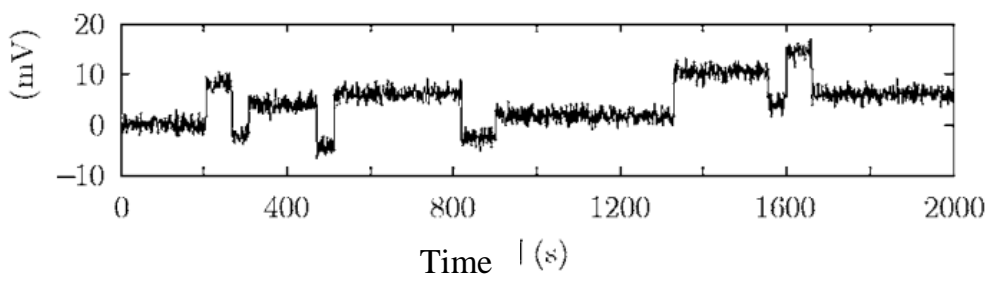

(a) non-stationary signal with noise

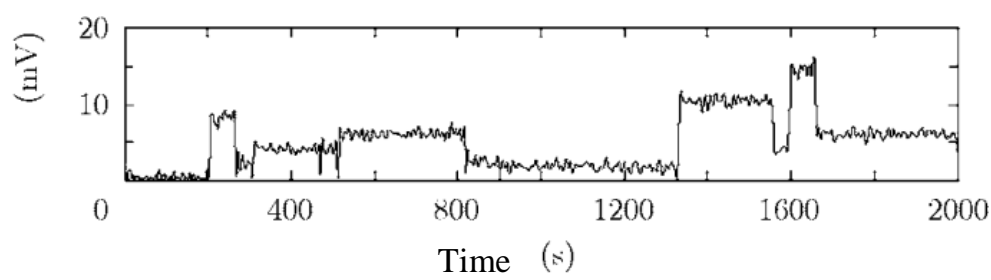

(b) Denoising result based on Fourier analysis

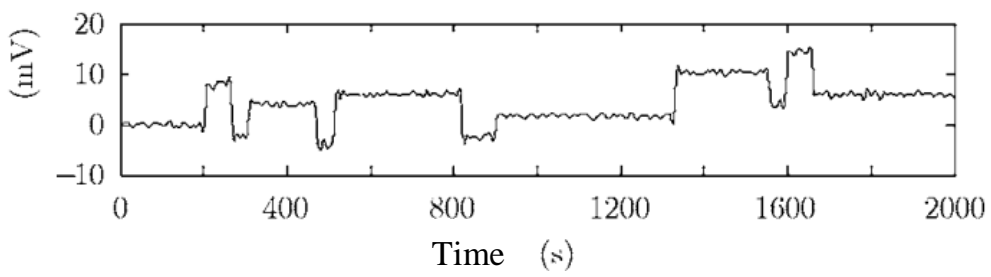

(c) Denoising result based on wavelet analysis

Figure 1. Fourier analysis of non-stationary signal analysis and wavelet filtering effect comparison chart 
From Fig.1(a), it can be seen that the useful signal is mainly concentrated in the low-frequency part, and the noise is mainly concentrated in the high-frequency part. Since the Fourier transform can not distinguish between the useful signal in the high frequency part and the noise, it can be observed from Fig.1(b) that the Fourier transform does not achieve good effect in noise reduction. And in Fig. 1 (c), the denoising method based on wavelet analysis has made achievement, and also keeps the information of mutation signal at the same time.

\section{EMI test system}

EMI block diagram of the test is shown in Fig.2. $\mathrm{S} 1[\mathrm{n}]$ is the signal received by the antenna 1 , which includes the useful electromagnetic signal $\mathrm{d}[\mathrm{n}]$ and the ambient electromagnetic interference a1[n]; a2[n] is the ambient electromagnetic signal received by the antenna 2; and $\mathrm{e}[\mathrm{n}]$, the output signal, which is used as the input signal of adaptive filtering ${ }^{[9]}$ to provide feedback and adjust the filter coefficient, outputs the useful electromagnetic signal finally, denoted by $\mathrm{d}[\mathrm{n}]$ due to errors.

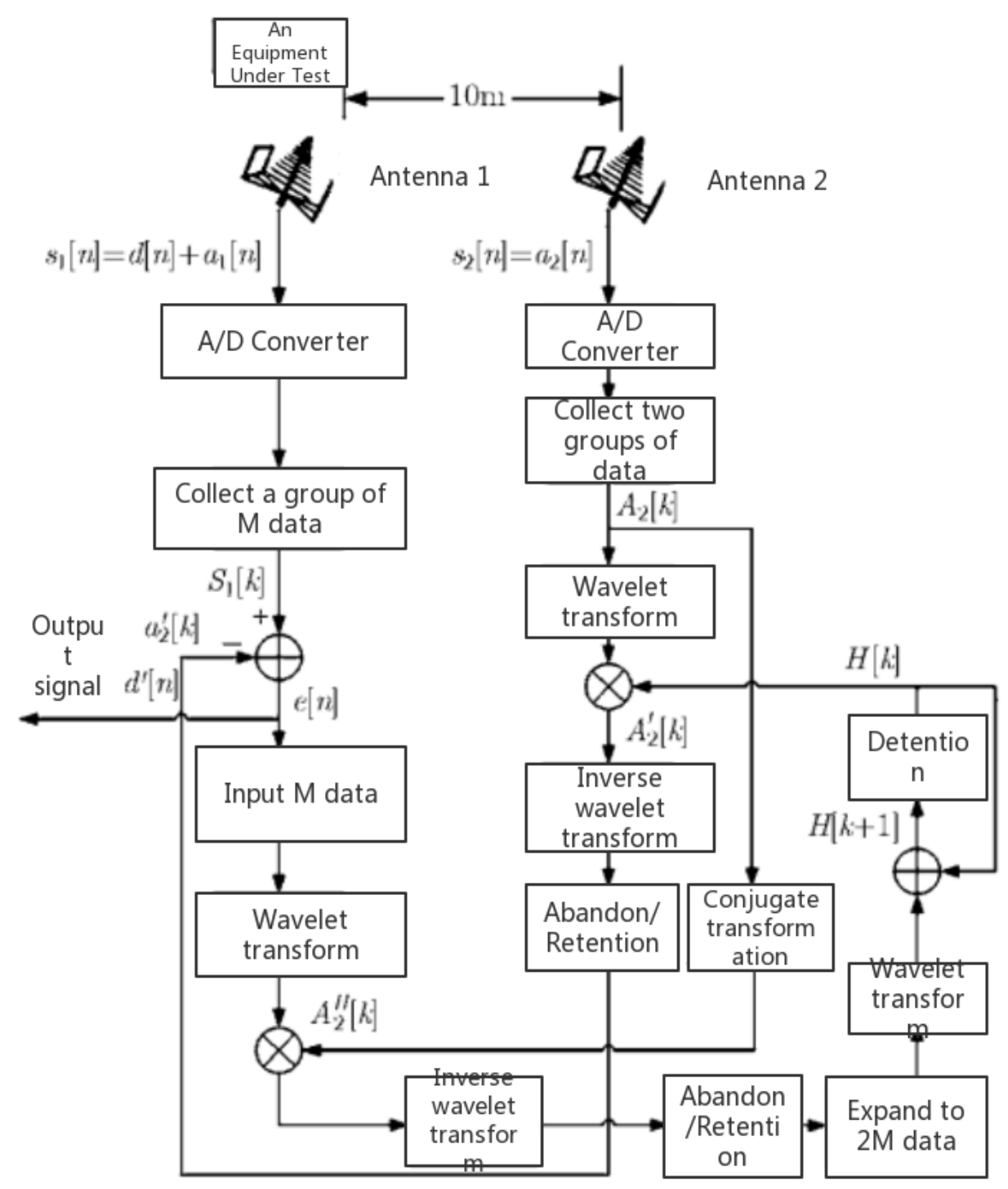

Figure 2. EMI block diagram of the test based on wavelet transform

\subsection{Signal decomposition}


Using the wavelet transform to decompose the signal acquired after $\mathrm{A} / \mathrm{D}$ converting. The images of the
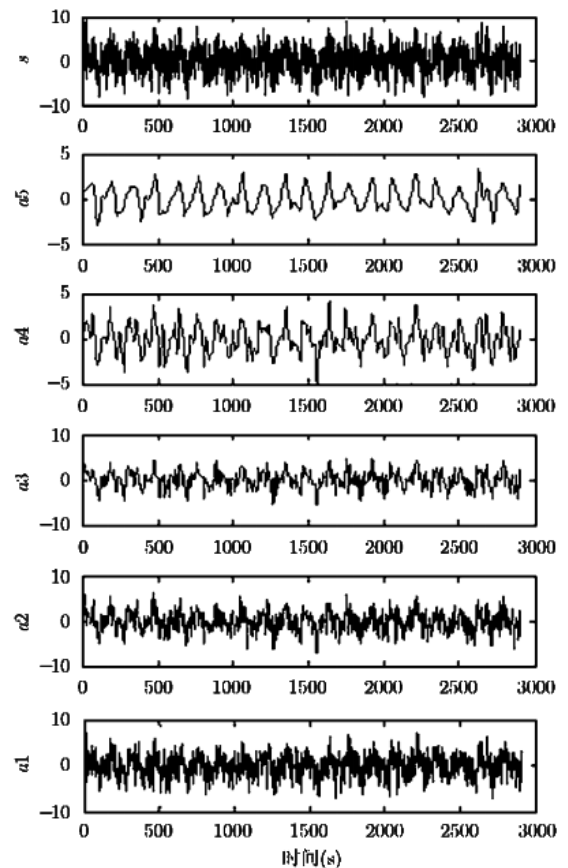
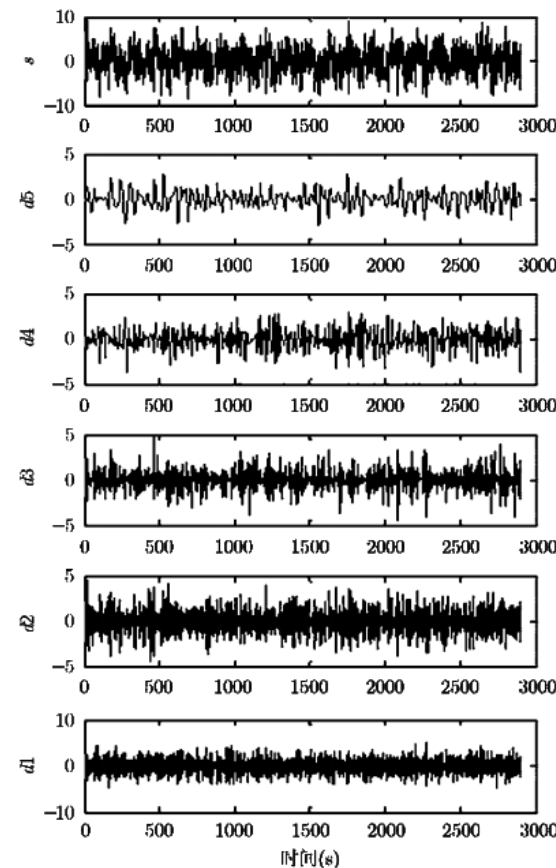

(a) The low frequency part of the original signal $\quad$ (b) The high frequency part of the original signal

Figure 3. The low and high frequency of signal after decomposition

From the figure, we can know that the d1 layer is the high frequency part of the signal, and its amplified signal diagram is shown in Figure3(a). The d4 layer includes the intermediate frequency part of the signal. While there appear discontinuous points in a3 and a4, because both the layers express the intermediate frequency signal.

Part of the information of the $\mathrm{d} 4$ layer is eliminated by the $\mathrm{d} 3$ layer, so the information of a1 a3 layer is needed to estimate the intermediate frequency signal. The low-frequency part of the signal can be seen from the a5 layer.

If the maximum frequency is 1 , the frequency bands of different signal layers are
$a 1: \quad 0-0.5$,
$d 1: 0.5^{-1}$
a2: $0-0.25$,
$d 2: \quad 0.25-0.5$;
a3: $0-0.125$,
d3: $0.125-0.25$;
a4: $0-0.0625$,
$d 4: \quad 0.0625-0.125$;
$a 5: \quad 0-0.03125$,
$d 5: \quad 0.03125-0.025$;

Therefore, the signal frequency bands can be analyzed after wavelet decomposition.

\subsection{Threshold filtering}

This paper uses the threshold function proposed in Section 1.2 to filter the signal. Wavelet transform can identify the abrupt information of non-stationary signal by decomposing the signal, and the electromagnetic interference from the surrounding environment is poor at the fine-scale layer, so in order to enhance the energy of the signal at the singularity point and restrain the interference energy, we multiply the wavelet coefficients of the neighboring fine-scales, and normalize the results to obtain a spatial shielding filter, and multiply it by the wavelet transform of the thinnest layer to acquire a new wavelet transform.

The two signals obtained are used as threshold. And then, the signal $\mathrm{S}[\mathrm{n}]$ is translated $(\mathrm{Phs}) \mathrm{i}=\mathrm{s}(\mathrm{i}+\mathrm{h}) \bmod \mathrm{n}$ to compare its wavelet coefficients with the corresponding thresholds, and the values below the threshold are filtered. Because the Morlet wavelet used in this paper has translation invariance, the translation denoising does not change the original signal. The process of signal wavelet denoising for $\mathrm{n}$ times is shown in equation (9), (10), $\bar{T}$ is reverse translation and Ave represents average.

$$
\begin{aligned}
& T\left(s,\left(P_{h}\right)_{h \in H_{n}}\right)=\operatorname{Ave}_{h \in H_{a}} P_{-h}\left(\bar{T}\left(P_{h} s\right)\right) \\
& H_{n}=\{h \mid 0 \leq h \leq n\}
\end{aligned}
$$




\subsection{The reconstruction of signal}

The reconstruction of signal is the inverse process of decomposition, and it can be obtained by iteration $^{[10]}$.

\section{Simulation and experimental verification}

\subsection{Comparison of simulation results}

This paper takes the switching power supply as an example to simulate and verify the method presented in the paper. The signal in Figure 4 is an actual switching power supply electromagnetic signal which is acquired after the filter of the mixed electromagnetic signal of the switching power supply with ambient interference by using the system designed in this paper. Figure 5 shows the contrast before and after wavelet denoising. And the dotted line represents the filtered electromagnetic radiation signal, and the full line represents the mixed signal received by the antenna 1 . By comparing figures 4 and 5, it can be found that the interference in FM, GSM, $100 \mathrm{MHz} 200 \mathrm{MHz}$ and $400 \sim 500 \mathrm{MHz}$ is all filtered out and the signal-noise ratio is increased $20 \sim 30 \mathrm{~dB}$. In addition, there is a $40 \mathrm{~dB}$ increase in signal-noise ratio of fixed signals and a $10 \mathrm{~dB}$ improvement in signal-noise ratio in the $210-230 \mathrm{MHz}, 575-590 \mathrm{MHz}$, and $680-760$ $\mathrm{MHz}$ bands. Not only the ambient electromagnetic interference noise in each frequency band is weakened, but also the spectrum of the radiation signal of the device is preserved.

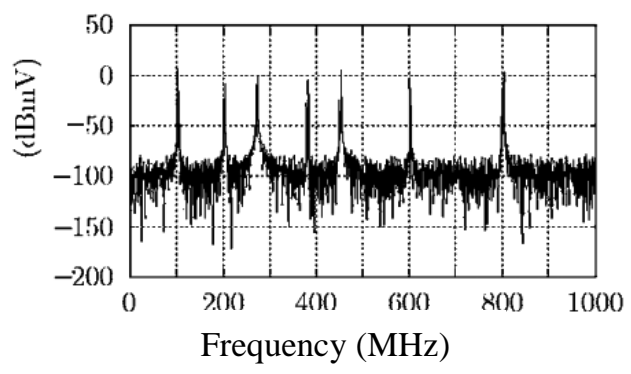

Figure 4. Spectrum map of the actual EMI signal of switching power supply

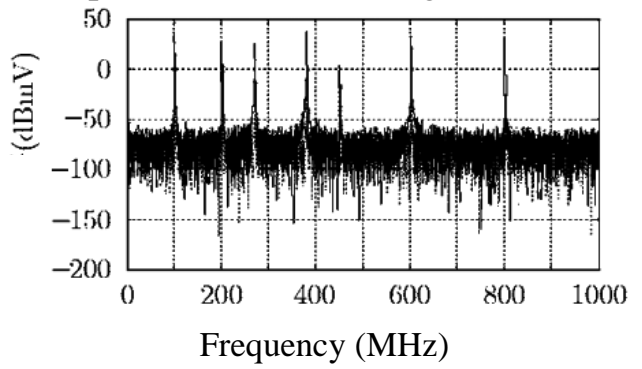

Figure 5. Spectrum map of signal mixed noise

\subsection{Comparison of the experimental results}

In order to verify the application of the system designed in this paper, we will use it to measure the electromagnetic interference of equipment, figure 6 and Figure 7 show the comparison.

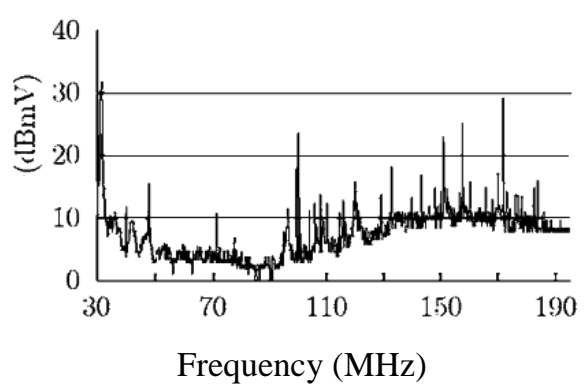

Figure 6. The result before filter 


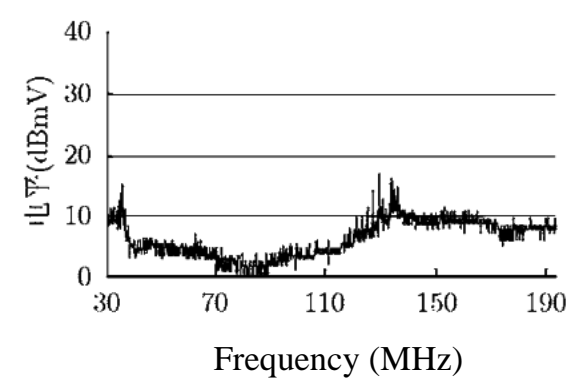

Figure 7. The result after filter

From the comparison, it can be seen that the threshold filtering EMI noise measurement method based on wavelet analysis can remove the environmental interference signal well and keep the electromagnetic signal of the device. And the interference signal of electronic devices from 30 $100 \mathrm{MHz}$ and signals of broadcast from $100 \sim 180$ $\mathrm{MHz}$ are successfully filtered.

\section{Conclusion}

Aiming at the problem that it is difficult to measure the electromagnetic radiation produced by the equipment with accuracy at present, this paper proposes a method for threshold filtering noise of EMI noise measurement based on wavelet analysis. This method can filter interference signals in environment effectively and rapidly at a large scale, which is especially useful in the filtering of non-stationary signals. Simulation and experimental results show that the system designed in this paper can do well in eliminating or attenuating the noise in the frequency range from $30 \mathrm{~Hz}$ to $1000 \mathrm{MHz}$ and is equipped with certain value in engineering application.

\section{References}

[1] Shinuzuka $T$ and Sugiura A. Reduction of ambient noise in EMI measurement [C]. IEEE International Symposium On Electromagnetic Compatibility Digest, Nagoya, Japan, 1989, September 8-10: 24-28.

[2] Parhami P, Marino M, Watkins S, and Nakauchi E. Innovative precompliance test methodology using ambient cancellation and coherence detection techniques[C]. IEEE International Symposium On Electromagnetic Compatibility, Seattle, USA, 1999: 1022-1025.

[3] Braun S, Krug F, and Russer P. A novel automatic digital quasipeak detector for a time-domain measurement system[C]. 2004 IEEE International Symposium On Electromagnetic Compatibility Digest, Santa Clara, USA, 2004: 832-837.

[4] Braun S, Al-Qedra M, and Russer P. A novel realtime time-domain measurement system based on field programmable gate arrays[C]. 17th International Zurich Symposium On Electromagnetic Compatibility, Singapore, 2006: 501-504.

[5] Frech A, Zakaria A, Braun S, and Russer P. Ambient noise cancelation with a time-domain EMI measurement system using adaptive filtering[C]. 2008 Asia-Pacific Sympsoium on Electromagnetic Compatibility \&19th International Zurich Symposium on Electromagnetic Compatibility, Singapore,

2008: 534-537.

[6] Klein A, Sauer T, Jedynak A, and Skrandies W. Conventional and wavelet coherence applied to sensory-evoked electrical brain activity [J]. IEEE Transactions on Biomedical Engineering, 2006, 53(2): 266-272.

[7] Yue Zhao and Niu Wen-cheng. The application of wavelet analysis in ultrasonic sensor system characteristic signal pretreament[J]. Acta Scientiarun Naturaltium Universitatis Nankaiensis, 2005, 38(2): 5-9.

[8] Zhang Hao, Blackburn T R, Phung B T, and Sen D. A novel wavelet transform technique for nn-line partial discharge measurements, IEEE Transactions on Dielectrics and Electrical Insulation. 2007, 14(1): 3-14.

[9] Huang Juan, Qian Xin, and Wang Cheng-lin. The construction of a wavelet filter and its application in environmental research[J]. Journal of Nanjing University (Natural Sciences), 2007, 43(4): 389-396.

[10] Ren Shou-xin and Gao Ling. Application of a wavelet packet transform based radial basis function neural network to analyze overlapping spectral[C]. 2008 Congress on Image and Signal Processing. China, 2008: 874-878. 